\title{
Hyaluronan-CD44 Interaction Regulates Mouse Retinal Progenitor Cells Migration, Proliferation and Neuronal Differentiation
}

Jian Ma ( $\nabla$ jian_ma@zju.edu.cn )

Zhejiang University School of Medicine Second Affiliated Hospital

\section{Xiaoyun Fang}

Zhejiang University School of Medicine Second Affiliated Hospital

Min Chen

Zhejiang University School of Medicine Second Affiliated Hospital

Yao Wang

Zhejiang University School of Medicine Second Affiliated Hospital

Li Zhang

Zhejiang University School of Medicine Second Affiliated Hospital

\section{Research Article}

Keywords: Retinal progenitor cells, Migration, Proliferation, Differentiation, Hyaluronan-CD44

Posted Date: November 10th, 2021

DOI: https://doi.org/10.21203/rs.3.rs-1057034/v1

License: (c) (i) This work is licensed under a Creative Commons Attribution 4.0 International License. Read Full License 


\section{Abstract}

Background: Therapeutic applications of retinal progenitor cells (RPCs) are hindered by their limited proliferation and differentiation capacity and poor ability to migrate into damaged retinal tissue. Our study aimed to explore the effects of HA-CD44 interactions on the regulation of RPCs migration, proliferation and differentiation, and to investigate the underlying regulation mechanisms.

Methods: Mouse RPCs were isolated and amplified. Western blot and flow cytometry analyses were used to investigate the expression of CD44 in RPCs. The effects of HA-CD44 interactions on the RPCs behaviors, including migration, proliferation and differentiation, were investigated by MTT assay, CCK8 assay, vertical collagen gel invasion assay, time-lapse imaging, immunocytochemistry, RT-PCR and western blot assay. Furthermore, the downstream signals of HA-CD44 interactions were investigated.

Results: CD44 was expressed in RPCs, and HA-CD44 interaction markedly improved RPCs adhesion and migration. The stimulation of miR-21 expression by HA-CD44 interaction was PKC/Nanog-dependent in RPCs. Treatment of RPCs with PKC- or Nanog-specific ASODN or miR-21 antagomir effectively blocked HA-mediated RPCs adhesion and migration. Moreover, ROK/Gab-1 associated PI3K/AKT signaling activation was required in the HA-CD44 interaction mediated RPCs proliferation and neuronal differentiation.

Conclusions: Our findings demonstrated new roles for HA-CD44 interaction in regulating both migration, proliferation and neuronal differentiation of RPCs. HA-CD44 signaling could comprise a novel approach to control RPC fates, which may be instructive for the application of RPCs for future therapeutic application.

\section{Background}

Retinal degeneration (RD), such as age-related macular degeneration and retinitis pigmentosa, is one of the major causes for irreversible blindness[1]. Although pharmacological treatments and gene therapies can delay the disease process, these treatments are unlikely to rescue the retinal neuron cells involved in retinal degeneration. Nowadays, stem cell transplantation therapies have shown great potential because of their abilities to replace the dying retinal neuron cells and preserve vision[2, 3]. Among them, retinal progenitor cells (RPCs) capable of self-renewal and differentiation into various retinal cell types have been brought into focus for sight restoration[4, 5]. RPCs were discovered in the adult mammalian eye and successfully isolated from the human retina, which can restore impaired visual function without tumorigenicity and ethical concerns $[4,6]$. However, the limited migration ability to the targeted position and lower differentiation capacity of RPCs toward specific retinal neurons in vivo hinders their future clinical applications[7]. Therefore, it is extremely important to explore mechanisms controlling RPCs migration and differentiation.

Hyaluronic acid ( $\mathrm{HA})$ is the simplest glycosaminoglycan and a major component of the extracellular matrix (ECM)[8]. Our recent study showed chondroitinase ABC facilitated the migration of mouse RPCs 
via disruption of the glial barriers. It may due to activation of the HA/CD44 signaling pathway to exert the effect[9]. Accumulating evidence demonstrates that HA plays a role in many facets of stem cell biology[10]. Previous studies have shown that HA would support mouse RPCs growth in vitro and in vivo[8]. HA not only regulates cell adhesion and motility, but also mediates cell proliferation and differentiation[8]. CD44, a major cell surface receptor for HA, is a family of multifunctional transmembrane glycoproteins and is expressed in numerous cells and tissues, including stem cell[11]. As reported, CD44 can connect the extracellular matrix to the cellular cytoskeleton and coordinate multiple downstream signaling pathways[11-13]. Recent studies have indicated that HA-CD44 interaction promotes both growth and invasion of head and neck squamous cell carcinoma[11]. However, the role of HA-CD44 interaction in the regulation of RPCs migration, proliferation and differentiation remains unknown.

In this study, the role of HA-CD44 interaction in the migration, proliferation and differentiation of RPCs was investigated. We observed that CD44 was expressed in mouse RPCs and further identified HA-CD44 interaction increased RPCs migration via PKC/Nanog/miR-21 signaling and promoted proliferation and retinal neuronal differentiation of RPCs via ROK/Gab-1 and PI3K/AKT signaling. Our findings provide important new insights into understanding the mechanisms regarding how HA-CD44 interaction regulate RPCs' migration, proliferation and differentiation and a new strategy to improve the repair result of RPCs in future therapeutic application of RD.

\section{Methods}

\section{RPCs isolation and culture}

According to our previous studies, RPCs were obtained from fresh retinal tissue of postnatal day 1 C57BL/ 6 mice, and were cultured with proliferation medium containing advanced Dulbecco's modified Eagle's medium (DMEM)/F12 (Invitrogen, Carlsbad, CA, USA), $20 \mathrm{ng} / \mathrm{ml}$ recombinant epidermal growth factor (EGF, Invitrogen), 2 mM L-glutamine (Invitrogen) and 1\% N2 neural supplement (Invitrogen)[9, 14]. For differentiation study, RPCs were cultured with differentiation medium containing advanced DMEM/F12 (Invitrogen), 10\% fetal bovine serum (FBS, Invitrogen), 1\% N2 neural supplement (Invitrogen) and without EGF. All animal experiments were approved by the Animal Ethics Committee of the Second Affiliated Hospital, School of Medicine, Zhejiang University and were performed in compliance with the ARRIVE guidelines.

\section{Drug treatment}

RPCs were treated with HA $(100 \mu \mathrm{g} / \mathrm{ml})$ for $72 \mathrm{~h}$ or CD 44 antibody $(10 \mu \mathrm{g} / \mathrm{ml})$ for $3 \mathrm{~h}$ followed by HA (100 $\mu \mathrm{g} / \mathrm{ml}$ ) for $72 \mathrm{~h}$ to assess the effect of HA-CD44 interaction on the cell migration, proliferation and differentiation. For knockdown experiments, cells were transfected with antisense oligonucleotides (ASODNs) or sense oligonucleotides (SODNs), miR-21 inhibitor or negative control. 


\section{Transfection}

ASODNs and SODNs were synthesized with a phosphorothioate backbone, purified with ULTRAPAGE (Sangon, Inc. Shanghai, China). For the transient transfection, the RPCs were treated with appropriate concentration of ODNs, miR-21 inhibitor, or negative control using lipofectamine 2000 Reagent (Invitrogen) according to manufacturer's instruction for 4 hours. Then the medium was removed and replaced with proliferation or differentiation medium.

\section{Cell adhesion assay}

The cell adhesion assay was performed as previously described with several modifications[15]. The 96well plates were treated with matrigel $(0.04 \mathrm{mg} / \mathrm{mL})$ (BD Biosciences, San Jose, CA) overnight to facilitate cell attachment. The different groups of RPCs were trypsinized and added to each well and allowed to attach for $2 \mathrm{~h}$, and then were washed gently with PBS twice. MTT solution $(5 \mathrm{mg} / \mathrm{mL})$ was added to the cells, and plates were further incubated at $37^{\circ} \mathrm{C}$ for $4 \mathrm{~h}$. The supernatant was carefully removed, and dimethyl sulfoxide (DMSO; Sigma-Aldrich, St. Louis, MO) was added to dissolve formazan crystals. The optical density was read on a spectrophotometer (Sunrise RC, Tecan, Switzerland) through a $490 \mathrm{~nm}$ filter. Cell adhesion rates $=(O D$ of the treated group cells $/ O D$ of the control group cells $) \times 100 \%$.

\section{Western blot analysis}

Proteins were isolated from the cultured RPCs, which were extracted in RIPA solution (Beyotime, Shanghai, China) with a protease inhibitor cocktail (Roche) and their concentrations were determined by the BCA protein assay kit (Beyotime; Beijing, China). Next, an equal amount of protein $(50 \mu \mathrm{g})$ from each sample was separated via sodium dodecyl sulfate-polyacrylamide gel electrophoresis (SDS-PAGE), and then transferred to polyvinylidene difluoride (PVDF) membranes (Millipore; Billerica, MA, USA). The membranes were blocked and incubated with primary antibodies against the following molecules overnight at $4^{\circ} \mathrm{C}$ : HA, PKC, $\beta$-tubulin (Abcam), CD44, Nanog, elF4A, survivin, PDCD4, $\beta$-actin (Proteintech), MDR1, XIAP (Abgent), ROK, p-Gab-1, Gab-1, p-AKT, AKT, cyclin D1 (Affinity), Hes1 (Saierbio) and GAPDH. The bands were detected with a chemiluminescence reagent and imaged by the ChemiDoc MP System (Bio-Rad; Hercules, CA, USA). The bands' intensities were quantified using Image Laboratory (version 2.0) software.

\section{Flow cytometry}

At passages 2 and 4, $1 \times 10^{6}$ RPCs were collected. CD44 expression on the RPCs was evaluated by flow cytometry using fluorescein conjugated HA (FL-HA) as previously described[16]. 


\section{Vertical collagen gel invasion assay}

The vertical collagen gel invasion assay was performed as previously described[17]. Monocyte chemoattractant protein-1 (MCP-1) was poured in the first layer as chemotactic reagent, and human umbilical vein endothelial cells (HUVECs) labeled with cell tracker red dye (Invitrogen, Carlsbad, CA, USA) were seeded onto the top surface of collagen gel to form a confluent monolayer. The different groups of RPCs labeled with cell tracker green dye (Invitrogen, Carlsbad, CA, USA) were added onto HUVECs and incubated at $37^{\circ} \mathrm{C}$ in $5 \% \mathrm{CO}_{2}$ in vertical position. The migration of cells was measured as the maximum distance from the surface of the collagen gel under fluorescent microscopy.

\section{Time-lapse video microscopy}

During the time-lapse recording, the different groups of RPCs were kept in a $37^{\circ} \mathrm{C}$ chamber with a $5 \% \mathrm{CO}_{2}$ atmosphere. To analyze migration behavior, serial phase-contrast images were captured with an inverted microscope (Zeiss Axiovert 200M) at $30 \mathrm{~s}$ intervals. The images were built into a movie using the Metamorph software.

\section{Immunocytochemistry}

At determined time, the different groups of RPCs were fixed with $4 \%$ paraformaldehyde (PFA). After blocking with $10 \%$ normal goat serum (Sigma-Aldrich), the cells were incubated with one of the following primary antibodies: Ki67, Nestin, GFAP, $\beta$-III-tubulin, Recoverin, Rhodopsin (Abcam), Crx (Omnimabs) $4{ }^{\circ} \mathrm{C}$ overnight. Then they were labeled with the corresponding secondary antibodies: Alexa 488-conjugated anti-mouse, Cy3-conjugated anti-mouse or FITC-conjugated anti-rabbit antibodies (Jackson). Finally, cells were counterstained with DAPI nuclear stain and observed by fluorescent microscope (Leica, Germany). The positive ratio was calculated with (immunepositive cells/DAPI stained cells in the field) $\times 100 \%$ by ImageJ software.

\section{Cell proliferation assay}

The cell proliferation assay was performed using the cell counting kit (CCK-8, Dojindo, Kumamoto, Japan) according to the instructions[18]. The different groups of RPCs were incubated with CCK-8 solution for 4 hours. The absorbance at $450 \mathrm{~nm}$ was read using a spectrophotometer (ELX800, BioTek, Vermont, USA).

\section{Reverse transcription and quantitative polymerase chain reaction (qPCR)}


Total RNA was extracted using Trizol Reagent (Invitrogen) and reverse transcribed according to manufacturer's instructions. Then the qPCR was performed using specific primers (Table 1). Relative miRNA expression was normalized to that of $U 6\left(2^{-\Delta C t}\right)$.

Table 1

qPCR primers for miRNAs

\begin{tabular}{|ll|}
\hline miRNA & Primer \\
\hline mmu miR21 & TGCGGCTAGCTTATCAGACT \\
\hline mmu miR191 & AGCAGGTGCGGGGCGGCGAAA \\
\hline mmu miR universal & CCAGTCTCAGGGTCCGAGGTATTC \\
\hline mmu U6 & CTCGCTTCGGCAGCACA \\
\hline
\end{tabular}

\section{Statistical analyses}

Statistics were expressed as mean \pm SD. The data were statistically analyzed by the one-way analysis of variance (ANOVA) or Student's $t$-test, and $P \leq 0.05$ was considered statistically significant.

\section{Results}

\section{RPCs express CD44 in vitro}

To investigate the roles of CD44 in RPCs migration and differentiation, we first explored the expression of CD44 in RPCs by western blot and flow cytometry analyses. As shown in Fig. 1, western blot analysis showed that CD44 was detected in the P2, P3, P4 RPCs, with more significant expression in the P3 RPCs. This result was strongly confirmed by flow cytometry analysis, CD44 expression in the P3 RPCs was significantly greater than its expression in the P2 or P4 RPCs. These findings strongly indicated that RPCs express CD44 in vitro.

\section{HA-CD44 interaction increases RPCs adhesion and migration via PKC/Nanog/miR-21 signaling}

HA-CD44 interaction plays a pivotal role in cytoskeleton activation and cell migration[19]. Previous studies indicated that HA/CD44-mediated PKC signaling regulates the stem cell marker (Nanog)associated miR-21 production, which in turn down-regulates the tumor suppressor protein (PDCD4) and promotes oncogenesis, leading to survivin, X-linked inhibitor of apoptosis protein (XIAP) and MDR1 expression. Survivin, XIAP and MDR1 play key roles in cell survival, expansion, and migration[20]. In this 
study we focused on whether HA-CD44 interaction regulates RPCs migration via PKC/Nanog/miR-21 signaling. To confirm the involvement of HA-CD44 interaction and PKC/Nanog/miR-21 signaling in RPCs adhesion and migration, $\mathrm{CD} 44$ or $\mathrm{PKC} / \mathrm{Nanog} / \mathrm{miR}-21$ signaling was inhibited. We first determined the effect of PKC- and Nanog-specific ASODNs on PKC and Nanog protein levels. As expected, PKC and Nanog protein levels were significantly decreased in PKC- and Nanog-specific ASODN-transfected cells compared with the control SODN-transfected cells (Fig. 2A and 2B).

The results of an MTT assay for cell adhesion are shown in Fig. 2C. The adhesion rate was greater in RPCs treated with HA than control or pretreated with anti-CD44 antibody followed by HA addition. Further analyses indicate that RPCs treated with PKC-specific ASODN or Nanog-specific ASODN display a decreased adhesion rate in the presence of HA. We also noted that downregulation of miR-21 by treating RPCs with miR-21 antagomir induced a significant decline in the cell adhesion rate of RPCs in the presence of HA. Cell migration assay was conducted using a transendothelial assay and time-lapse imaging. Our data showed that RPCs treated with HA exhibited better ability to migrate than control or RPCs pretreated with anti-CD44 antibody followed by HA addition (Fig. 2D and 2E). We also noted that RPCs treated with PKC-specific ASODN or Nanog-specific ASODN or miR-21 antagomir display decreased ability to migrate in the presence of HA (Fig. 2D and 2E). Time-lapse imaging results were consistent with the results shown above (showed in Supplementary Material).

To determine whether miR-21 levels are upregulated following the binding of HA to CD44, our results indicate that the level of miR-21 is increased in RPCs treated with HA (Fig. 3A) compared with control or those cells pretreated with anti-CD44 antibody followed by HA treatment (Fig. 3A). These findings suggest that HA-CD44 interaction has an important role in the production of miR-21 in RPCs. Furthermore, RPCs treated with PKC-specific ASODN or Nanog-specific ASODN show significantly less HA-induced miR-21 expression than SODN groups (Fig. 3A). These findings support the notion that both PKC and Nanog are required for miR-21 production in HA-activated RPCs. In addition, we have found that the expression of miR-21 can be induced in RPCs treated with miR-21 antagomir NC upon addition of HA (Fig. 3A). In contrast, the treatment of RPCs with miR-21 antagomir plus HA results in a decrease in miR21 expression (Fig. 3A). Moreover, there were no significant difference among the levels of the miR-191 control in all samples (Fig. 3B).

Finally, we explore HA/CD44-mediated PKC/Nanog/miR-21 signaling on the expression of MDR1, elF4A, survivin, XIAP and PDCD4. Our results indicate that the expression of PKC, MDR1, elF4A, survivin and XIAP were significantly increased in RPCs treated with HA compared with RPCs treated with no HA (Fig. 3C) or in those cells pretreated with anti-CD44 antibody followed by HA addition (Fig. 3C). In contrast, HA treatment promoted down-regulation of the PDCD4 expression in RPCs (Fig. 3C). Furthermore, we have observed that the expression of MDR1, elF4A, survivin and XIAP were significantly inhibited when RPCs were pretreated with PKC-specific ASODN or Nanog-specific ASODN but not SODN followed by HA addition (Fig. 3C), respectively. We have also confirmed that down-regulation of miR-21 by antagomir promotes down-regulation of MDR1, elF4A, survivin and XIAP in the presence of HA (Fig. 3C). However, the expression of PDCD4 was significantly increased when PKC/Nanog/miR-21 signaling was inhibited. 


\section{HA-CD44 interaction promotes proliferation and retinal neuronal differentiation of RPCs via ROK/Gab-1 and PI3K/AKT signaling}

ROK/Gab-1 and PI3K/AKT are key signaling pathways in HA/CD44-mediated cellular functions, such as proliferation and cell survival[13]. Previous work has also indicated a positive link between ROK/Gab-1 associated PI3K/AKT signaling activation during HA/CD44-mediated breast cancer progression[13]. In this study we focused whether HA-CD44 interaction regulates proliferation and retinal neuronal differentiation of RPCs via ROK/Gab-1 and PI3K/AKT signaling. To confirm the involvement of HA-CD44 interaction and ROK/Gab-1 and PI3K/AKT signaling in RPCs proliferation and differentiation, CD44 or ROK/Gab-1 and PI3K/AKT signaling was inhibited. We first determined the effect of Gab-1- and ROKspecific ASODN on Gab-1 and ROK protein levels. As expected, Gab-1 and ROK protein levels were significantly decreased in Gab-1- and ROK-specific ASODN-transfected cells compared with the control SODN-transfected cells (Fig. 4A).

The results of the CCK8 assay showed that the cell proliferations of RPCs treated with HA were significantly increased than control or pretreated with anti-CD44 antibody followed by HA addition (Fig. 4B). Further analyses indicate that RPCs treated with ROK-specific ASODN, Gab-1-specific ASODN or PI3K inhibitor LY294002 display a decreased proliferation rate in the presence of HA (Fig. 4B). At the same time, the immunofluorescence assay results showed that the increased expression levels of Ki67 in RPCs treated with HA than control or pretreated with anti-CD44 antibody followed by HA addition (Fig. 4C and 4D). Similarly, the expression levels of Ki67 were reduced in RPCs treated with ROK-specific ASODN, Gab1-specific ASODN or LY294002 (Fig. 4C and 4D). These data indicated that HA-CD44 interaction regulates proliferation of RPCs via ROK/Gab-1 and PI3K/AKT signaling.

Further study was focused on the effects of HA-CD44 interaction on RPC differentiation.

Immunofluorescence analysis showed that the RPCs differentiation markers, such as glial fibrillary acidic protein (GFAP, the astrocyte marker), and $\beta$-III-tubulin (a pan-neuronal marker), cone-rod homeobox (Crx, the cone-rod photoreceptor precursor marker), recoverin (the cone and rod photoreceptor marker) and rhodopsin (the rod photoreceptor marker) were significantly increased in differentiation medium than in proliferation medium (Fig. 5C and 5D, Fig. 6-7). Meanwhile, we detected a prominent decrease of the progenitor marker nestin in differentiation medium (Fig. 5A and 5B). When the RPCs were treated with HA, the levels of retinal neuronal cell markers ( $\beta$-III-tubulin, Crx, recoverin, rhodopsin) were significantly increased, while GFAP and nestin were significantly downregulated (Fig. 5-7). Further analyses indicate that RPCs treated with anti-CD44 antibody, ROK-specific ASODN, Gab-1-specific ASODN or PI3K inhibitor LY294002 had the opposite effect in the presence of HA (Fig. 5-7). These results indicate that HA-CD44 
interaction enhances the differentiation of RPCs toward neuronal cells via ROK/Gab-1 and PI3K/AKT signaling.

Based on the above results, we further explored whether there is an interaction between ROK/Gab-1 and $\mathrm{PI} 3 \mathrm{~K} / \mathrm{AKT}$ signaling in HA/CD44-mediated RPCs proliferation. Our results indicate that the protein expressions of $\mathrm{p}-\mathrm{Gab}-1, \mathrm{p}-\mathrm{AKT}$ and the proliferation marker cyclin D1 were increased significantly in RPCs treated with HA than control or pretreated with anti-CD44 antibody followed by HA addition (Fig. 4E). Moreover, treatment of RPCs with ROK-specific ASODN results in down-regulation of the protein expressions of p-Gab-1, p-AKT and cyclin D1 in the presence of HA. Meanwhile, Gab-1-specific ASODN significantly reduced the protein expressions of p-AKT and cyclin D1 in the presence of HA (Fig. 4E). Furthermore, PI3K inhibitor LY294002 also greatly reduced the protein expressions of p-AKT and cyclin D1 in the presence of HA, while the expression level of p-Gab-1 had no obvious changes (Fig. 4E).

We also investigated the potential interaction between ROK/Gab-1 and PI3K/AKT signaling in HA/CD44mediated RPCs neuronal differentiation process. Western blot analysis revealed that RPCs treated with differentiation medium demonstrate a significant increase in expression of p-Gab-1, p-AKT and Hes1 compared to RPCs treated with proliferation medium (Fig. 8). However, the expression of the stem cell marker CD44 was significant reduced (Fig. 8). Further analyses indicate that HA treatment significantly increased the expressions of p-Gab-1 and p-AKT, and reduced the expressions of CD44 and Hes1 (Fig. 8). Meanwhile, RPCs treated with anti-CD44 antibody had the opposite effect in the presence of HA (Fig. 8). Moreover, treatment of RPCs with ROK-specific ASODN significantly reduced the protein expressions of $p$ Gab-1, p-AKT and increased the expressions of CD44 and Hes1 in the presence of HA (Fig. 8). Moreover, Gab-1-specific ASODN significantly reduced the protein expression of p-AKT and increased the expressions of CD44 and Hes1 in the presence of HA (Fig. 8). Furthermore, PI3K inhibitor LY294002 also greatly reduced the protein expression of p-AKT and increased the expressions of CD44 and Hes 1 in the presence of HA, while the expression level of p-Gab-1 had no obvious changes (Fig. 8).

These observations indicate that ROK/Gab-1 signaling is important upstream activator for PI3K/AKT signaling required for HA/CD44-mediated mediated RPCs proliferation and neuronal differentiation.

\section{Discussion}

To achieve successful RPC-based transplantation therapy for RD, high efficacy of cell migration to targeted position and differentiation into specific retinal neurons are two main challenges[7, 18]. HA not only serves as the primary ECM components but also acts as an active signaling molecule[11-13]. It is now well established that manipulations of $\mathrm{HA}$ concentration or interaction can significantly alter the activities of signaling pathways that are involved in the regulation of cell behaviors and oncogenesis[10]. CD44 is main receptor for HA that influence cell proliferation, survival and motility, and are known to be relevant to neural stem cells (NSCs) expansion and differentiation[21]. However, the research on HA-CD44 interaction in RPCs is rather scarce. As the retina belongs to the central nervous system, RPCs are thought to possess a multipotency similar to NSCs. Furthermore, CD44 was found to be highly expressed in RPCs 
in vitro in the present study. It is reasonable to hypothesize that HA-CD44 interaction might have effect on RPCs behaviors such as cell migration, proliferation and differentiation. In the present study, we reported that HA-CD44 interaction promotes the migration of RPCs, which might be due to the activation of $\mathrm{PKC} /$ Nanog/miR-21 signaling. Furthermore, our data suggested that HA-CD44 interaction allowed for RPCs proliferation and retinal neuronal differentiation by invoking ROK/Gab-1 signaling pathway, and subsequently activating the PI3K/AKT signaling pathway.

HA-CD44 interaction leads to numerous cellular responses, including those that involve tyrosine kinases, PKC, PI3K, as well as cytoskeletal components[13, 22]. The stem cell marker Nanog plays a key role in the self-renewal and maintenance of pluripotency in embryonic stem cells[23]. Disruption of the interaction between HA and CD44 in breast tumour cells have been shown to inhibit PKC-Nanog signaling mediated miR-21 production and suppress cell survival[10,23]. Previous studies have also demonstrated that miR21 is involved in the promotion of cell invasion, migration, and growth[23]. Consistent with these studies, our data demonstrated that PKC/Nanog/miR-21 signaling is involved in HA-CD44-mediated RPC migration. We found that HA could promote the migration of RPCs, whereas perturbing the interaction between HA and CD44 or suppressing PKC/Nanog/miR-21 signaling activity had an opposite effect on RPC migration. Growing evidence supports that IAP proteins (e.g. survivin and XIAP), MDR1 and elF4A positively modulate migration, invasion and metastasis[24]. In our study, we found that HA could enhance the expression of those proteins, whereas reduce the expression of PDCD4, which is closely linked to apoptosis and translation inhibition.

Moreover, our study also supports that HA-CD44 interaction may promote RPCs proliferation and retinal neuronal differentiation. In our attempts to identify the potential cell signaling pathway, we noticed that ROK/Gab-1 and PI3K/AKT are the two main pathways for cell proliferation and differentiation, which had been reported on for tumor cells and stem cells[25]. PI3K/AKT is known as not only an important proliferation-related signaling pathway, but also a differentiation-related signaling pathway in mesenchymal stem cells (MSCs)[26]. Moreover, previous study demonstrated that ROK/Gab-1 signaling acts as the upstream effector of PI3K/AKT signaling during HA/CD44-mediated cell functions[25]. Most importantly, a recent study demonstrated that the Gel-HA hydrogel markedly enhanced RPCs proliferation, while RPCs cultured with the Gel-HA-PDA hydrogel might be programmed to differentiate into neurons by interaction with PI3K/AKT signaling[7]. Our results are consistent with this report showing that HA markedly upregulated expressions of the retinal progenitor-related marker nestin and cell proliferation marker Ki-67 in RPCs in proliferation medium, while upregulated expressions of the differentiation markers in differentiation medium. Furthermore, we found that ROK/Gab-1 signaling is important upstream activator for PI3K/AKT signaling required for HA/CD44-mediated mediated RPCs proliferation and neuronal differentiation. In summary, our data suggested that HA-CD44 interaction allowed for RPCs proliferation and retinal neuronal differentiation by invoking ROK/Gab-1 signaling pathway, and subsequently activating the PI3K/AKT signaling pathway.

\section{Conclusion}


Our study provides novel insights into how HA-CD44 interaction regulates RPCs migration, proliferation and differentiation and further studies will focus on underlying the role of HA-CD44 interaction in retinal development and their application in vivo to treat RD.

\section{Abbreviations}

ANOVA: Analysis of variance; ASODNs: Antisense oligonucleotides; DMEM: Dulbecco's modified Eagle's medium; ECM: Extracellular matrix; EGF: Epidermal growth factor; GFAP: Glial fibrillary acidic protein; HA: Hyaluronic acid; HUVECs: Human umbilical vein endothelial cells; MCP-1: Monocyte chemoattractant protein-1; qPCR: quantitative polymerase chain reaction; PFA: Paraformaldehyde; PVDF: Polyvinylidene difluoride; RD: Retinal degeneration; RPCs: Retinal progenitor cells; SDS-PAGE: Sodium dodecyl sulfatepolyacrylamide gel electrophoresis; SODNs: Sense oligonucleotides.

\section{Declarations}

\section{Ethics approval and consent to participate}

All animal experiments were approved by the Animal Ethics Committee of the Second Affiliated Hospital, School of Medicine, Zhejiang University and were performed in compliance with the ARRIVE guidelines.

\section{Consent for publication}

Not applicable.

\section{Competing interests}

The authors declare no conflicts of interest.

\section{Funding}

This work was supported by the grant from the Natural Science Foundation of China (No.81571819); and by the Natural Science Foundation of Zhejiang Province, China (No.LY21H120002).

\section{Authors' contributions}

J.M., X.F., M.C., Y.W., and L.Z. conceived and designed the experiments. J.M., M.C., Y.W., and L.Z. performed the experiments. J.M. and X.F. analyzed the data. J.M. and X.F. wrote the paper. J.M., X.F., M.C., Y.W., and L.Z. reviewed and edited the manuscript. 


\section{Acknowledgements}

The authors are grateful to all the editors and reviewers for their profound insight.

\section{References}

1. Bourne RR, Stevens GA, White RA, Smith JL, Flaxman SR, Price H, Jonas JB, Keeffe J, Leasher J, Naidoo K et al: Causes of vision loss worldwide, 1990-2010: a systematic analysis. Lancet Glob Health 2013, 1(6):e339-349.

2. Jones MK, Lu B, Girman S, Wang S: Cell-based therapeutic strategies for replacement and preservation in retinal degenerative diseases. Prog Retin Eye Res 2017, 58:1-27.

3. Nazari H, Zhang L, Zhu D, Chader GJ, Falabella P, Stefanini F, Rowland T, Clegg DO, Kashani AH, Hinton DR et al: Stem cell based therapies for age-related macular degeneration: The promises and the challenges. Prog Retin Eye Res 2015, 48:1-39.

4. Klassen HJ, Ng TF, Kurimoto Y, Kirov I, Shatos M, Coffey P, Young MJ: Multipotent retinal progenitors express developmental markers, differentiate into retinal neurons, and preserve light-mediated behavior. Invest Ophthalmol Vis Sci 2004, 45(11):4167-4173.

5. Jiang $C$, Klassen $H$, Zhang $X$, Young $M$ : Laser injury promotes migration and integration of retinal progenitor cells into host retina. Mol Vis 2010, 16:983-990.

6. Li SY, Yin ZQ, Chen SJ, Chen LF, Liu Y: Rescue from light-induced retinal degeneration by human fetal retinal transplantation in minipigs. Curr Eye Res 2009, 34(7):523-535.

7. Tang Z, Jiang F, Zhang Y, Zhang Y, YuanYang, Huang X, Wang Y, Zhang D, Ni N, Liu F et al: Musselinspired injectable hydrogel and its counterpart for actuating proliferation and neuronal differentiation of retinal progenitor cells. Biomaterials 2019, 194:57-72.

8. Liu Y, Wang R, Zarembinski TI, Doty N, Jiang C, Regatieri C, Zhang X, Young MJ: The application of hyaluronic acid hydrogels to retinal progenitor cell transplantation. Tissue Eng Part A 2013, 19(12):135-142.

9. Ma J, Chen M, Ai J, Young MJ, Ge J: Enhanced migration of engrafted retinal progenitor cells into the host retina via disruption of glial barriers. Mol Vis 2021, 27:300-308.

10. Toole BP: Hyaluronan: from extracellular glue to pericellular cue. Nat Rev Cancer 2004, 4(7):528539.

11. Gomez KE, Wu F, Keysar SB, Morton JJ, Miller B, Chimed TS, Le PN, Nieto C, Chowdhury FN, Tyagi A et al: Cancer Cell CD44 Mediates Macrophage/Monocyte-Driven Regulation of Head and Neck Cancer Stem Cells. Cancer Res 2020, 80(19):4185-4198.

12. Bourguignon LY: Hyaluronan-CD44 interaction promotes microRNA signaling and RhoGTPase activation leading to tumor progression. Small GTPases 2012, 3(1):53-59.

13. Bourguignon LY: Matrix hyaluronan-activated CD44 signaling promotes keratinocyte activities and improves abnormal epidermal functions. Am J Pathol 2014, 184(7):1912-1919. 
14. Ma J, Kabiel M, Tucker BA, Ge J, Young MJ: Combining chondroitinase ABC and growth factors promotes the integration of murine retinal progenitor cells transplanted into Rho(-/-) mice. Mol Vis 2011, 17:1759-1770.

15. Zhang Y, Sun H, Chen X, Li J, Zhao H, Geng L, Li B: Functional profile of gastric epithelial cells infected with Helicobacter pylori strains. Microb Pathog 2016, 95:77-81.

16. Perschl A, Lesley J, English N, Trowbridge I, Hyman R: Role of CD44 cytoplasmic domain in hyaluronan binding. Eur J Immunol 1995, 25(2):495-501.

17. Cho Y, Lee SE, Lee HC, Hur J, Lee S, Youn SW, Lee J, Lee HJ, Lee TK, Park J et al: Adipokine resistin is a key player to modulate monocytes, endothelial cells, and smooth muscle cells, leading to progression of atherosclerosis in rabbit carotid artery. J Am Coll Cardiol 2011, 57(1):99-109.

18. Wang Y, Zhang D, Tang Z, Zhang Y, Gao H, Ni N, Shen B, Sun H, Gu P: REST, regulated by RA through miR-29a and the proteasome pathway, plays a crucial role in RPC proliferation and differentiation. Cell Death Dis 2018, 9(5):444.

19. Bourguignon LY, Gilad E, Peyrollier K, Brightman A, Swanson RA: Hyaluronan-CD44 interaction stimulates Rac1 signaling and PKN gamma kinase activation leading to cytoskeleton function and cell migration in astrocytes. J Neurochem 2007, 101(4):1002-1017.

20. Jiang G, Huang C, Liao X, Li J, Wu XR, Zeng F, Huang C: The RING domain in the anti-apoptotic protein XIAP stabilizes c-Myc protein and preserves anchorage-independent growth of bladder cancer cells. J Biol Chem 2019, 294(15):5935-5944.

21. Su W, Foster SC, Xing R, Feistel K, Olsen RH, Acevedo SF, Raber J, Sherman LS: CD44 Transmembrane Receptor and Hyaluronan Regulate Adult Hippocampal Neural Stem Cell Quiescence and Differentiation. J Biol Chem 2017, 292(11):4434-4445.

22. Wang SJ, Bourguignon LY: Role of hyaluronan-mediated CD44 signaling in head and neck squamous cell carcinoma progression and chemoresistance. Am J Pathol 2011, 178(3):956-963.

23. Bourguignon LYW, Spevak CC, Wong G, Xia W, Gilad E: Hyaluronan-CD44 Interaction with Protein Kinase C€ Promotes Oncogenic Signaling by the Stem Cell Marker Nanog and the Production of MicroRNA-21, Leading to Down-regulation of the Tumor Suppressor Protein PDCD4, Anti-apoptosis, and Chemotherapy Resistance in Breast Tumor Cells. Journal of Biological Chemistry 2009, 284(39):26533-26546.

24. Fulda S: Regulation of cell migration, invasion and metastasis by IAP proteins and their antagonists. Oncogene 2013, 33(6):671-676.

25. Bourguignon LY, Singleton PA, Zhu H, Diedrich F: Hyaluronan-mediated CD44 interaction with RhoGEF and Rho kinase promotes Grb2-associated binder-1 phosphorylation and phosphatidylinositol 3-kinase signaling leading to cytokine (macrophage-colony stimulating factor) production and breast tumor progression. J Biol Chem 2003, 278(32):29420-29434.

26. Chen J, Crawford R, Chen C, Xiao Y: The key regulatory roles of the PI3K/Akt signaling pathway in the functionalities of mesenchymal stem cells and applications in tissue regeneration. Tissue Eng Part $B$ Rev 2013, 19(6):516-528. 
Figures

A
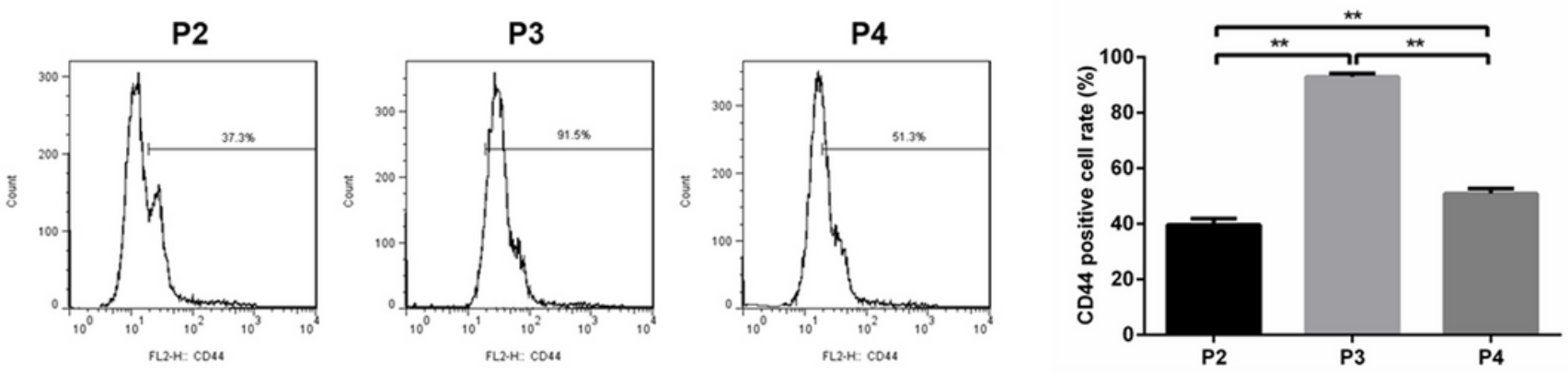

B
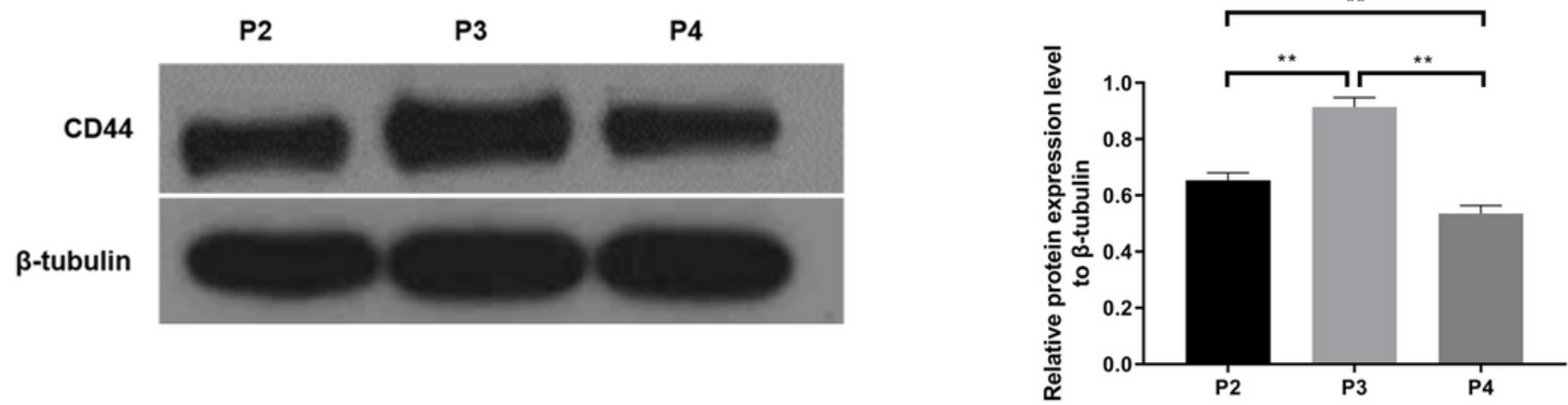

\section{Figure 1}

Expression of CD44 on RPCs in vitro. (A) Flow cytometry was used to analyze the CD44 expression from the P2 to the P4 RPCs. (B) Western blotting showed CD44 expressed from the P2 to the P4 RPCs. The data were shown as means $\pm \mathrm{SD}$, $* \star \mathrm{p}<0.01$. 
A

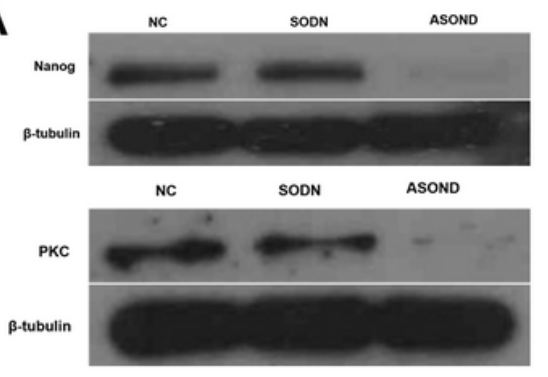

B

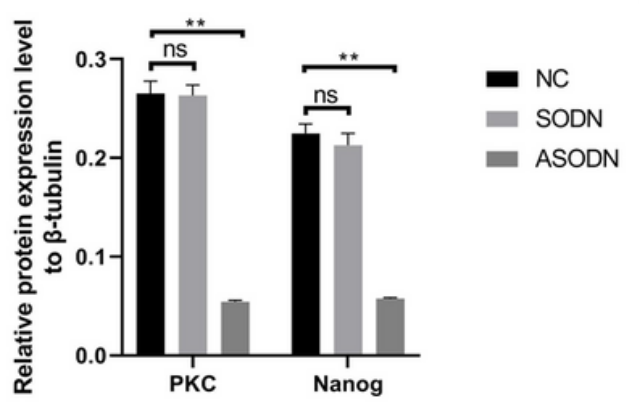

C

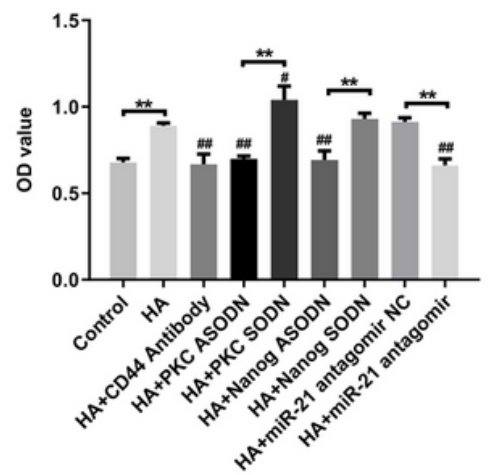

$E$

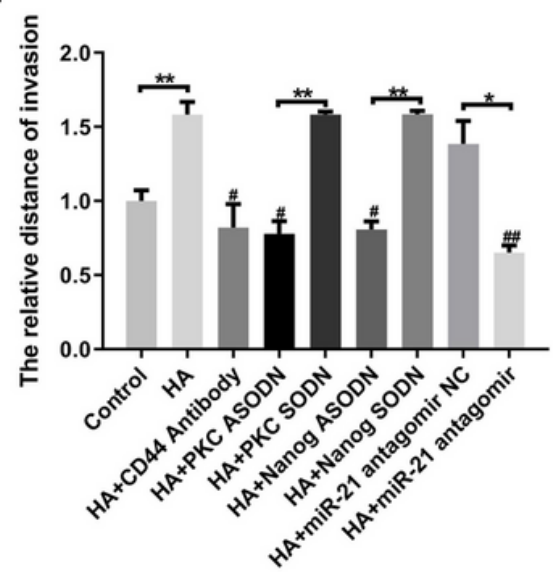

Figure 2

HA-CD44 interaction and PKC/Nanog/miR-21 signaling are closely linked to RPCs adhesion and migration. (A, B) The western blot results revealed that the expression levels of PKC and Nanog were significantly decreased in the PKC- and Nanog-specific ASODN-transfected cells compared with the control SODN-transfected cells. (C) The cell adhesion ability of the RPCs with different treatments was assessed via MTT analysis. (D, E) The cell migration ability of the RPCs with different treatments was assessed using transendothelial assay. The data were shown as means $\pm S D,{ }^{\star} p<0.05 ;{ }^{\star \star} p<0.01 ; \# p<$ $0.05, \# \# p<0.01$ versus HA group. 
A

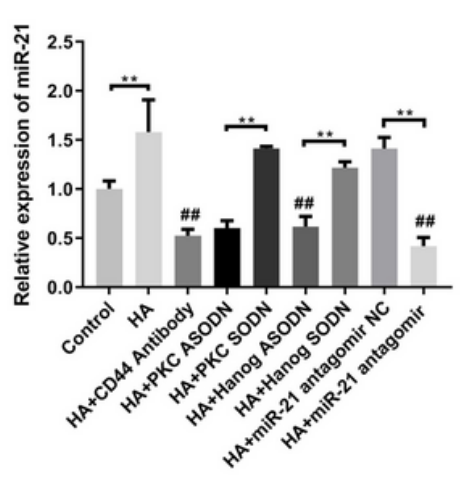

C
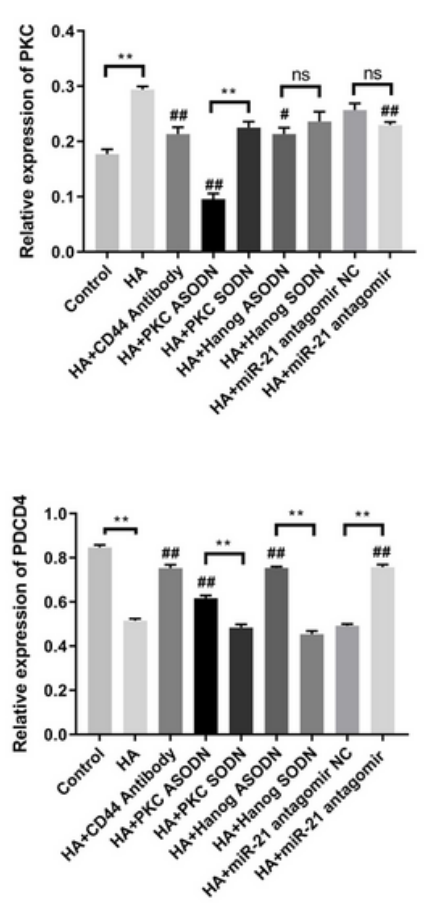

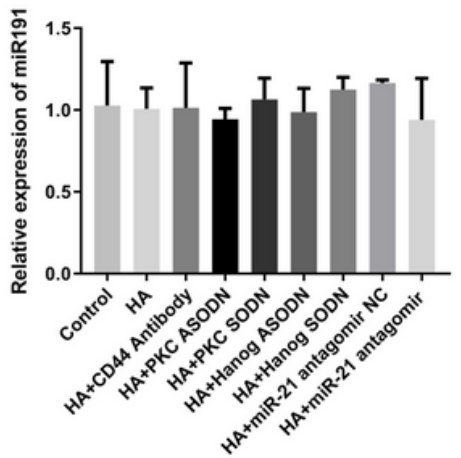

B

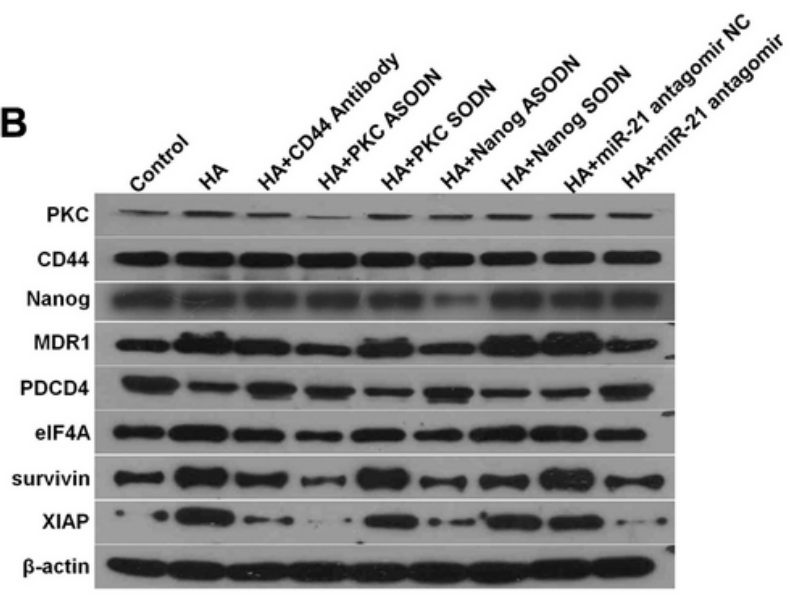

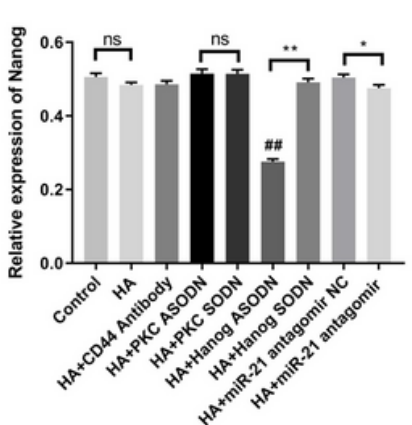

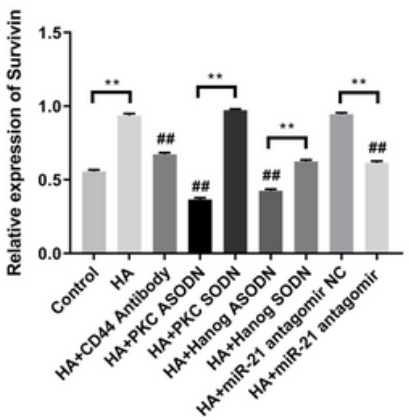

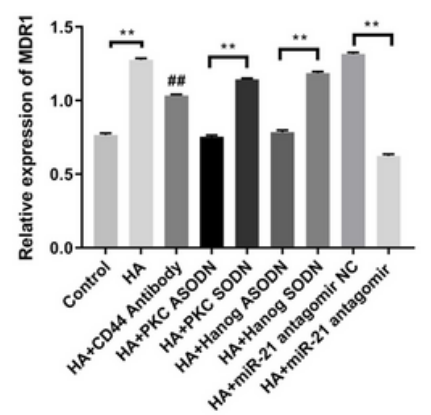
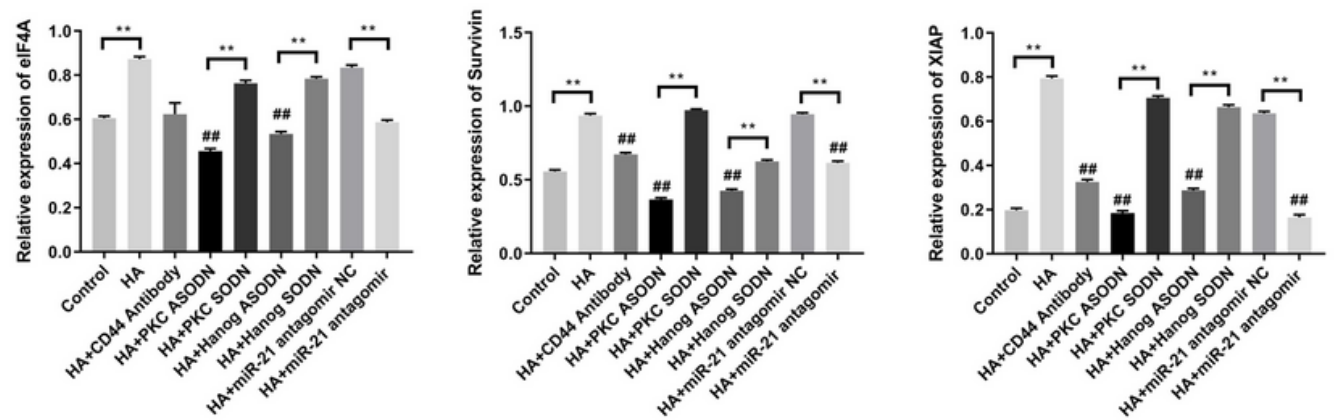

Figure 3

HA-CD44 interaction activates PKC/Nanog/miR-21 signaling and regulates the expression of migrationrelated proteins in RPCs. (A) The expression levels of miR-21 and miR-191 with different treatments were analyzed by qPCR. (B, C) The expression levels of CD44, PKC, Nanog and migration-related proteins with different treatments were analyzed by western blot. The data were shown as means $\pm S D,{ }^{*} p<0.05 ;{ }^{*} p<$ 0.01 ; \# $p<0.05$, \# $\mathrm{p}<0.01$ versus HA group. 
A
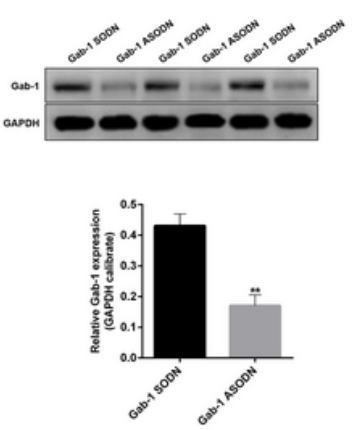

C
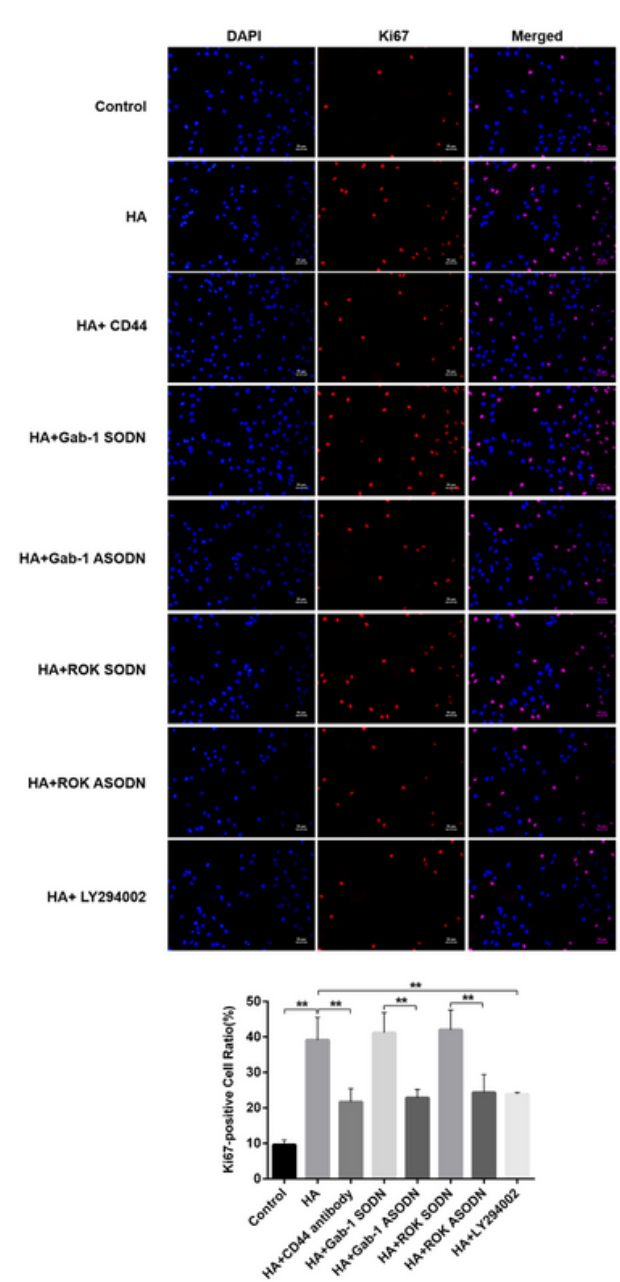

B

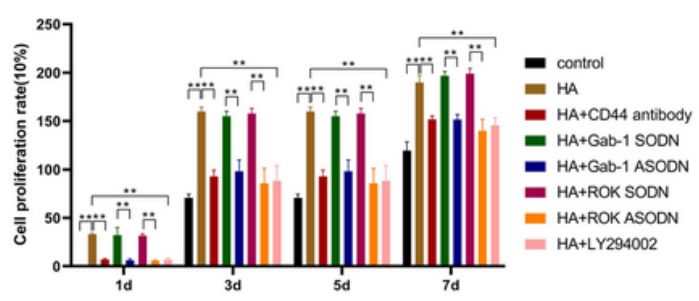

D
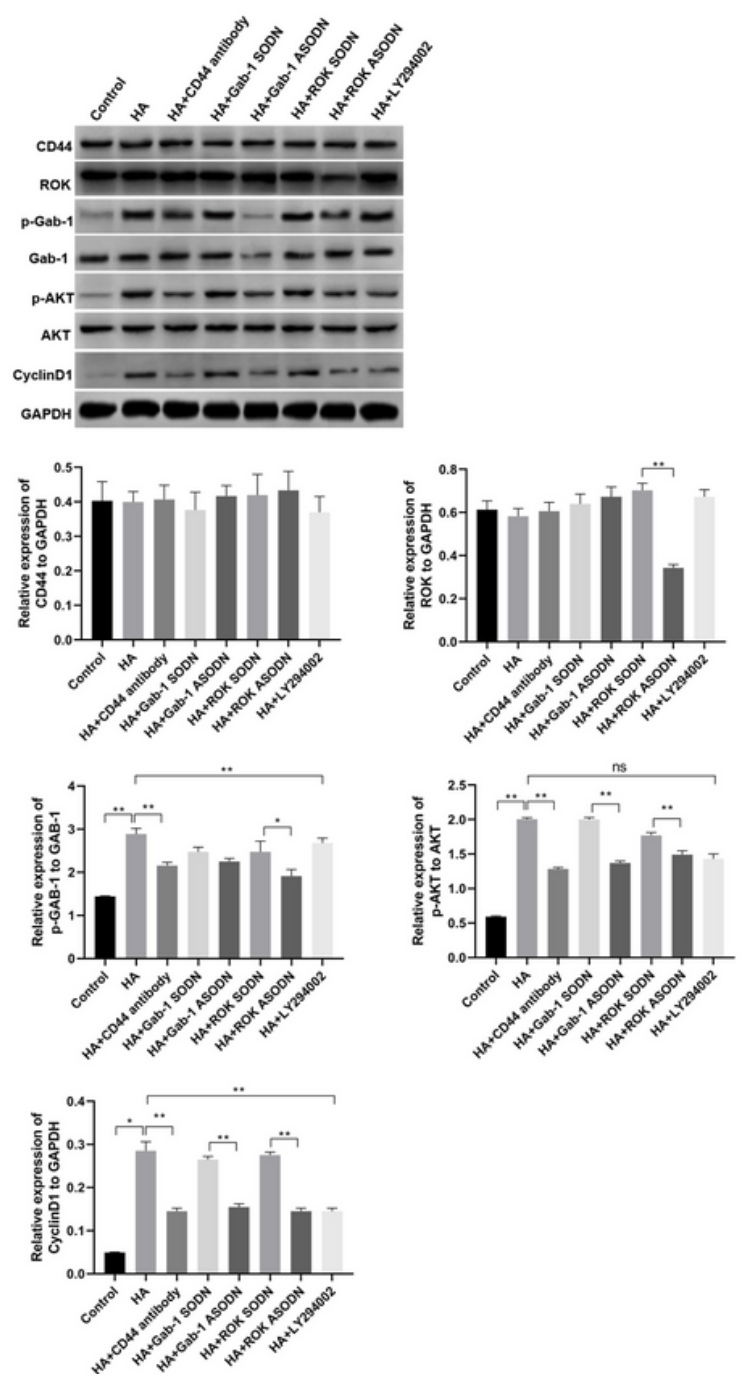

\section{Figure 4}

HA-CD44 interaction promotes RPCs proliferation via ROK/Gab-1 and PI3K/AKT signaling. (A) The western blot results revealed that the expression levels of Gab and ROK were significantly decreased in the Gab- and ROK-specific ASODN-transfected cells compared with the control SODN-transfected cells.

(B) Proliferation of RPCs with different treatments was analyzed by CCK8 assay. (C) Proliferation of RPCs with different treatments was analyzed by Ki67 immunofluorescence assay. (D, E) The expression levels of CD44, ROK, p- Gab-1, Gab-1, p-AKT, AKT and the proliferation marker cyclin D1 with different 
treatments were analyzed by western blot. The data were shown as means $\pm S D,{ }^{*} p<0.05 ;{ }^{*} p<0.01$; $\star \star \star p<0.001$; scale bar $=50 \mu \mathrm{m}$.

A

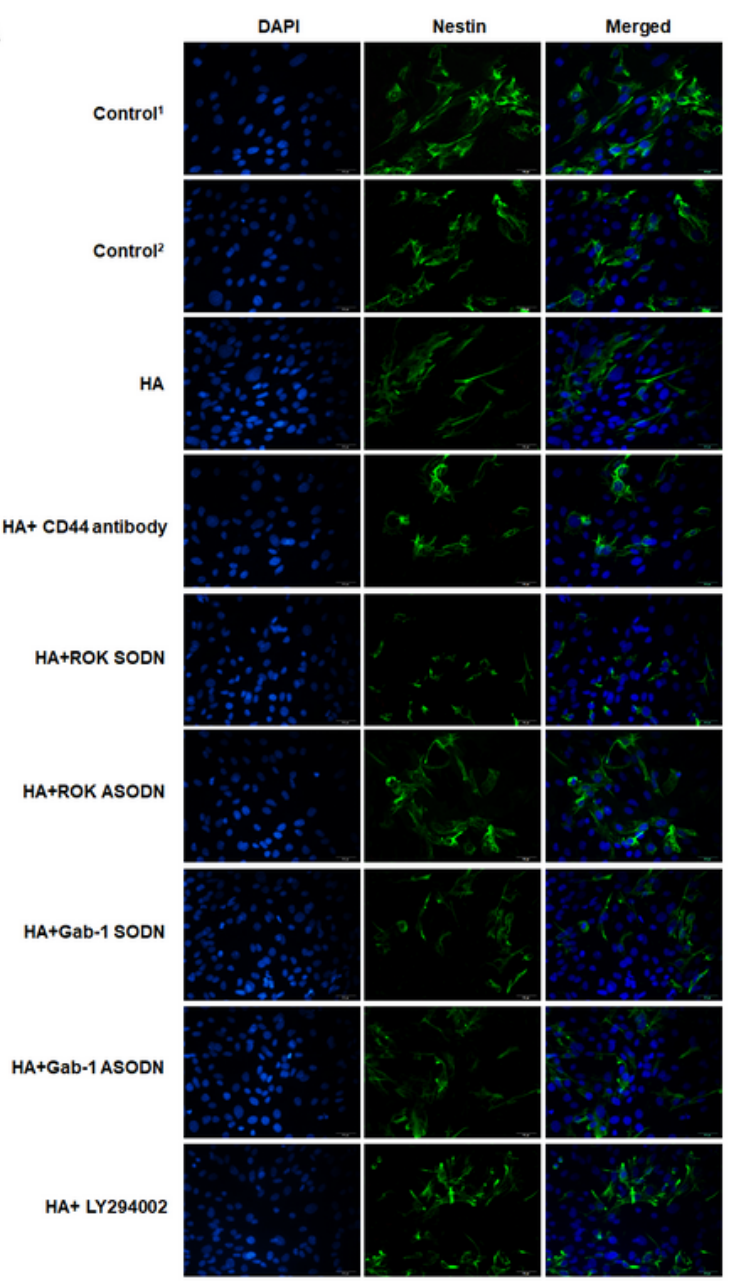

B

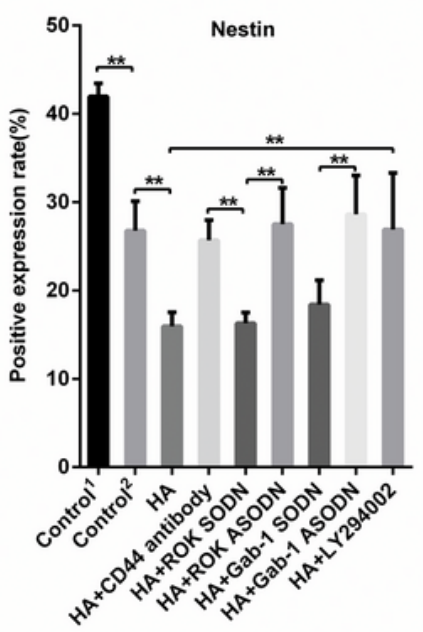

C

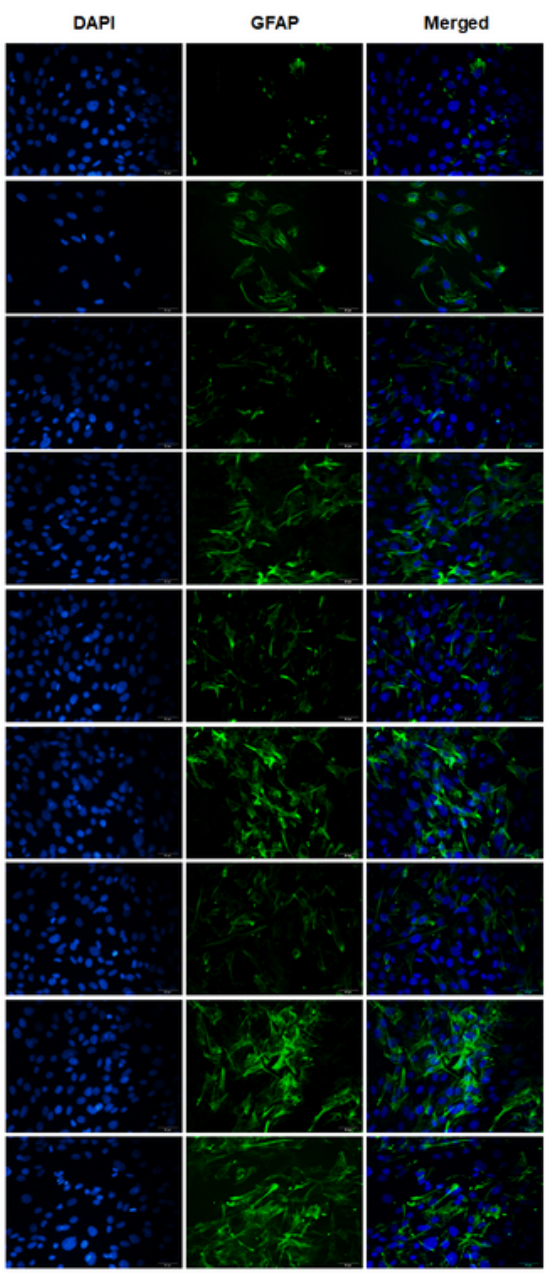

D

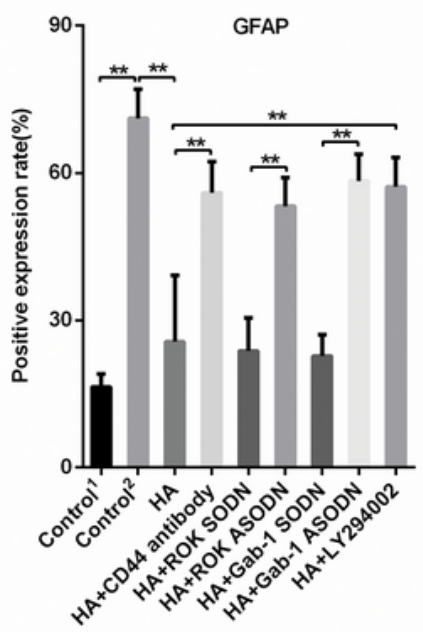

\section{Figure 5}

HA-CD44 interaction, ROK/Gab-1 and PI3K/AKT signaling inhibit the differentiation of RPCs toward glial cells. (A, B) The immunocytochemistry analysis was used to detect nestin-positive cells with different treatments. (C, D) The immunocytochemistry analysis was used to detect glial fibrillary acidic protein 
(GFAP)-positive cells with different treatments. The data were shown as means $\pm S D$, ${ }^{\star \star} p<0.01$; Scale bar $=20 \mathrm{~mm}$; Control1, proliferation medium, Control2, differentiation medium.

A

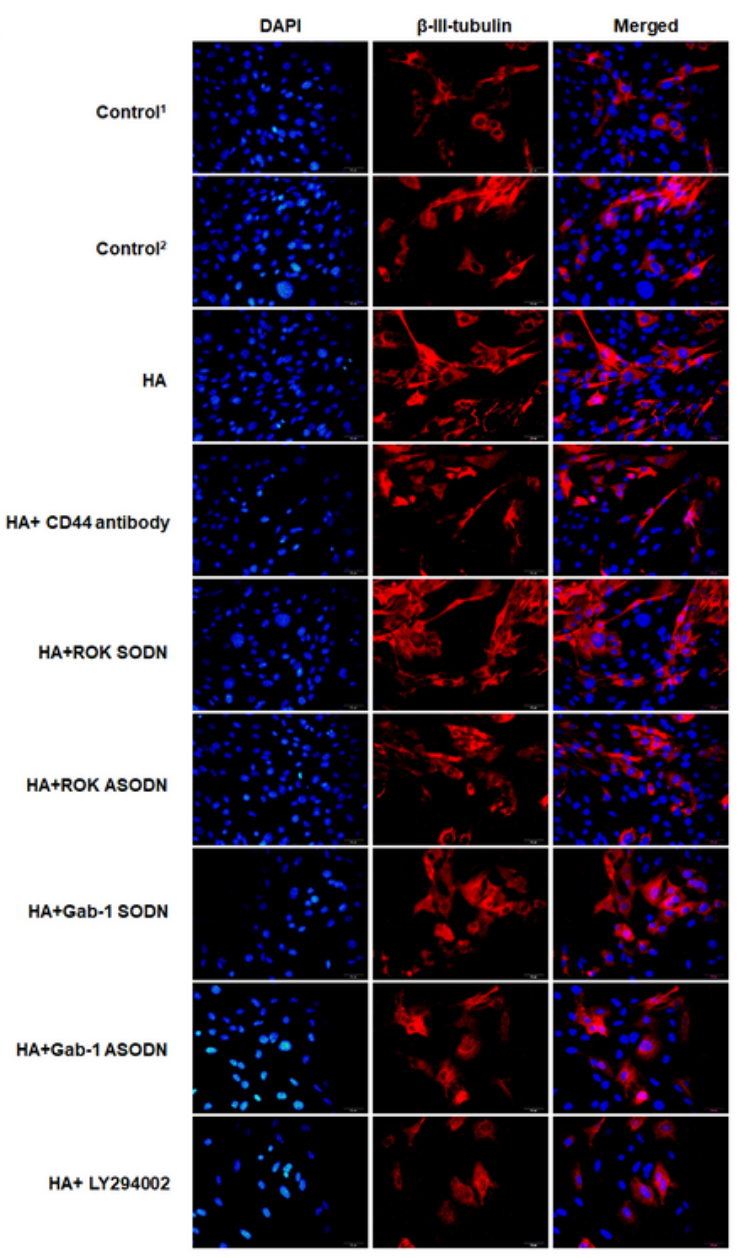

B

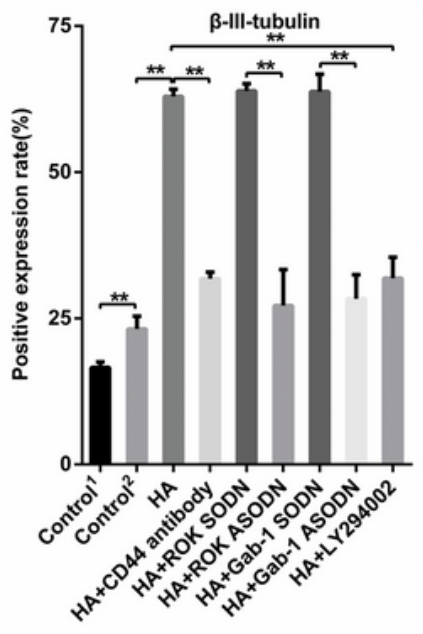

C

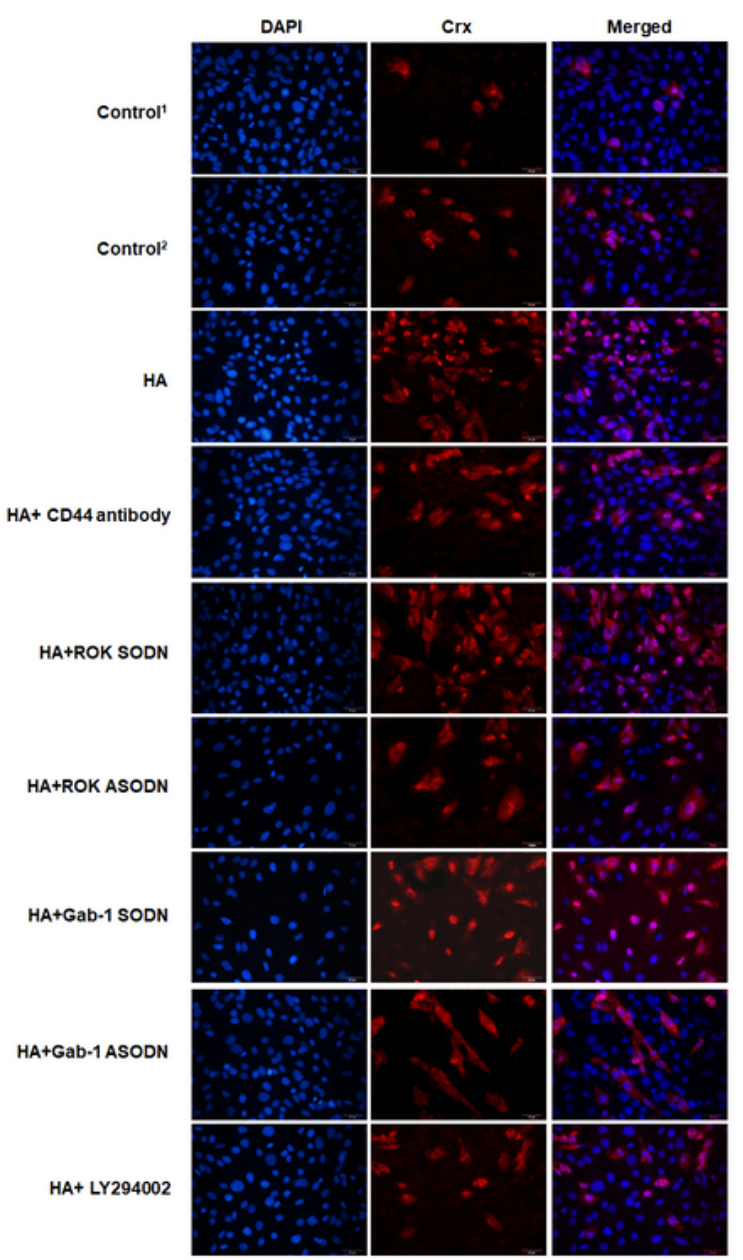

D

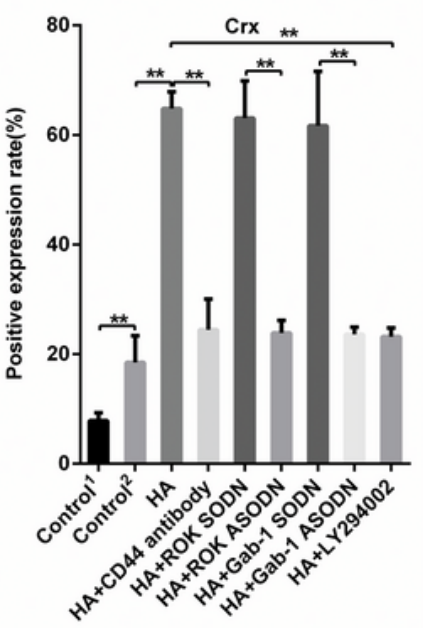

\section{Figure 6}

HA-CD44 interaction, ROK/Gab-1 and PI3K/AKT signaling enhance the differentiation of RPCs toward neuronal cells. (A, B) The immunocytochemistry analysis was used to detect $\beta$-III-tubulin-positive cells with different treatments. (C, D) The immunocytochemistry analysis was used to detect cone-rod 
homeobox (Crx)-positive cells with different treatments. The data were shown as means $\pm \mathrm{SD},{ }^{\star \star} \mathrm{p}<0.01$; Scale bar = $20 \mathrm{~mm}$; Control1, proliferation medium, Control2, differentiation medium.

A

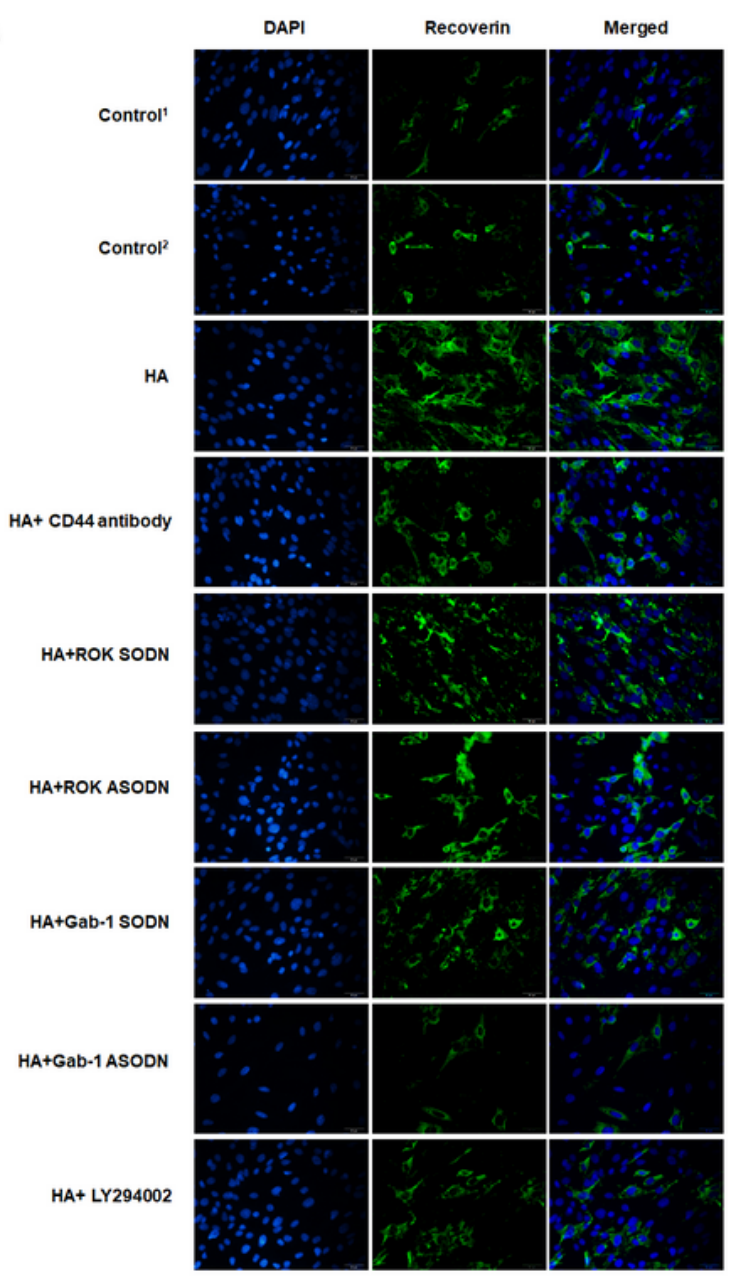

B

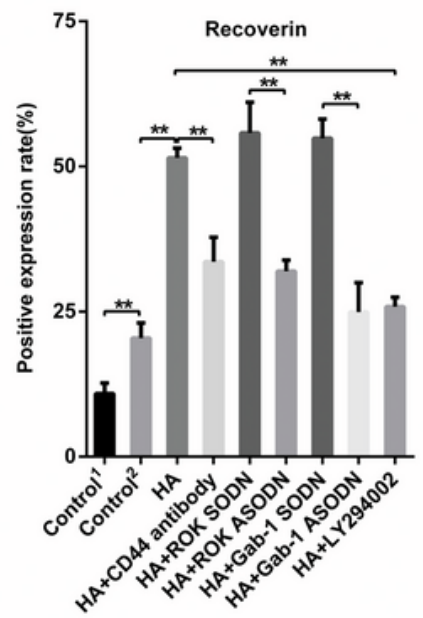

C

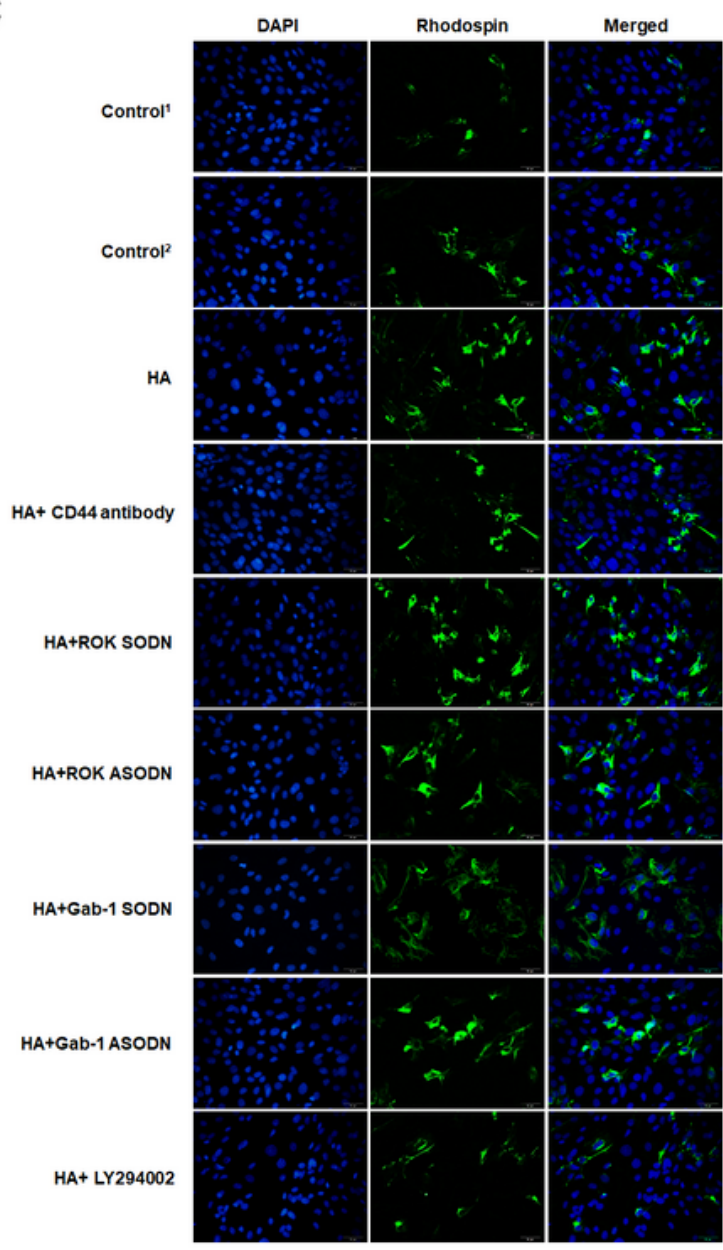

D

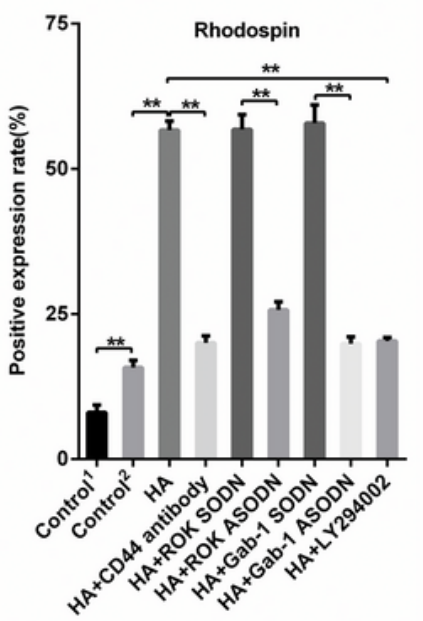

\section{Figure 7}

HA-CD44 interaction, ROK/Gab-1 and PI3K/AKT signaling enhance the differentiation of RPCs toward neuronal cells. (A, B) The immunocytochemistry analysis was used to detect recoverin-positive cells with different treatments. (C, D) The immunocytochemistry analysis was used to detect rhodopsin-positive 
cells with different treatments. The data were shown as means $\pm S D$, ${ }^{\star \star} \mathrm{p}<0.01$; Scale bar $=20 \mathrm{~mm}$; Control1, proliferation medium, Control2, differentiation medium.
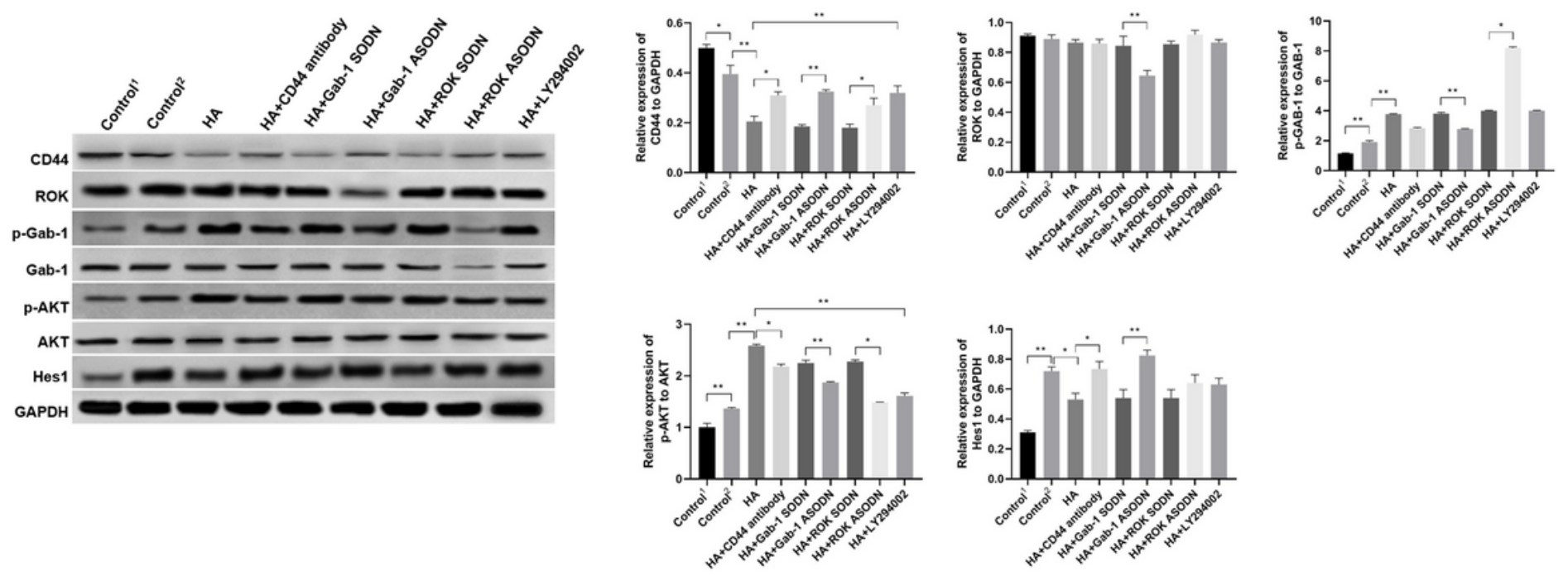

\section{Figure 8}

HA-CD44 interaction regulates the activation of the ROK/Gab-1/ PI3K/AKT axis. The expression levels of CD44, ROK, p- Gab-1, Gab-1, p-AKT, AKT and Hes1 with different treatments were analyzed by western blot. ${ }^{\star} \mathrm{p}<0.05 ;{ }^{* *} \mathrm{p}<0.01$.

\section{Supplementary Files}

This is a list of supplementary files associated with this preprint. Click to download.

- SupplementaryMaterial.mp4 